\title{
EULER CHARACTERISTICS OF COMPLETE INTERSECTIONS
}

\author{
JOHN EWING AND SURESH MOOLGAVKAR ${ }^{1}$
}

\begin{abstract}
We point out that a conjecture of Chen and Ogiue, regarding the Euler characteristic of complete intersections, is a simple consequence of a theorem of Hirzebruch.
\end{abstract}

Let $F_{1}, F_{2}, \ldots, F_{r}$ be nonsingular hypersurfaces of degrees $a_{1}, a_{2}, \ldots, a_{r}$ in complex projective space $\mathbf{C} P^{n+r}$, and suppose that these hypersurfaces are in general position. The intersection $F_{1} \cap F_{2} \cap \cdots \cap F_{r}$ is a nonsingular algebraic manifold denoted by $V_{n}\left[a_{1}, a_{2}, \ldots, a_{r}\right]$. In [1] it was conjectured that the Euler characteristic, $\chi\left(V_{n}\left[a_{1}, a_{2}, \ldots, a_{r}\right]\right)=n+1$ if and only if $a_{1} a_{2} \cdots a_{r}=1$ in case $n$ is even; and $\chi\left(V_{n}\left[a_{1}, a_{2}, \ldots, a_{r}\right]\right)=n+1$ if and only if either $a_{1} a_{2} \cdots a_{r}=1$ or $a_{1} a_{2} \cdots a_{r}=2$ in case $n$ is odd. In this short note we point out that this conjecture is a trivial consequence of the result of Hirzebruch [2].

THEOREM 1 (HIRZEBRUCH).

$$
\sum_{n=0}^{\infty} \chi\left(V_{n}\left[a_{1}, a_{2}, \ldots, a_{r}\right]\right) z^{n}=\frac{a_{1} a_{2} \cdots a_{r}}{(1-z)^{2}} \prod_{i=1}^{r} \frac{1}{1+\left(a_{i}-1\right) z} .
$$

REMARK. Clearly, $a_{1} a_{2} \cdots a_{r} \mid \chi\left(V_{n}\left[a_{1}, a_{2}, \ldots, a_{r}\right]\right)$.

We note that we have explicit expressions in the 2 cases:

$$
\begin{aligned}
\chi\left(V_{n}[a]\right) & =n+2+\left((1-a)^{n+2}-1\right) / a, \\
\chi\left(V_{n}[2,2]\right) & =2\left(1+(-1)^{n}\right) .
\end{aligned}
$$

LEMMA 2.

$$
\begin{aligned}
& (-1)^{n} \chi\left(V_{n}\left[a_{1}, a_{2}, \ldots, a_{r}\right]\right) \\
& \quad=a_{r} \sum_{k=0}^{n}\left(a_{r}-1\right)^{n-k}(-1)^{k} \chi\left(V_{k}\left[a_{1}, a_{2}, \ldots, a_{r-1}\right]\right) .
\end{aligned}
$$

Proof. This follows immediately by multiplying power series and Theorem 1. Q.E.D.

LEMMA 3. If $a_{1} a_{2} \cdots a_{r}>2$ then $(-1)^{n} \chi\left(V_{n}\left[a_{1}, a_{2}, \ldots, a_{r}\right]\right) \geqslant 0$.

Received by the editors August 25, 1975.

AMS (MOS) subject classifications (1970). Primary 57D20, 14M10.

Key words and phrases. Euler characteristic, complete intersection manifold, complex projective space.

${ }^{1}$ Partially supported by a fellowship from the Office of Research and Development, Indiana University. 
Proof. Using Lemma 2 inductively, the statement reduces to the fact that

$$
(-1)^{n} \chi\left(V_{n}[2,2]\right) \geqslant 0 \text { and }(-1)^{n} \chi(V[a]) \geqslant 0 \text { for } a>2 \text {. }
$$

Q.E.D.

LEMMA 4. If $a_{1} a_{2} \cdots a_{r-1}>2$ then

$$
(-1)^{n} \chi\left(V_{n}\left[a_{1}, a_{2}, \ldots, a_{r}\right]\right) \geqslant(-1)^{n} a_{r} \chi\left(V_{n}\left[a_{1}, a_{2}, \ldots, a_{r-1}\right]\right) .
$$

Proof. By Lemmas 2 and $3,(-1)^{n} \chi\left(V_{n}\left[a_{1}, a_{2}, \ldots, a_{r}\right]\right)$ is given as a sum of positive terms, the last of which is $(-1)^{n} a_{r} \chi\left(V_{n}\left[a_{1}, a_{2}, \ldots, a_{r-1}\right]\right)$. Q.E.D.

CoRollary 5. If $\chi\left(V_{n}\left[a_{1}, a_{2}, \ldots, a_{r}\right]\right)=n+1$, then one of the following two cases obtains:

(i) $n$ is even and $a_{1} a_{2} \cdots a_{r}=1$.

(ii) $n$ is odd and either $a_{1} a_{2} \cdots a_{r}=1$ or $a_{1} a_{2} \cdots a_{r}=2$.

PRoof. Suppose that $n$ is even and $a_{1} a_{2} \cdots a_{r} \geqslant 2$. Then by the remark following Theorem $1, a_{i}>2$ for each $i$. Applying Lemma 4 inductively, we have for some $a>2$,

$$
n+1=\chi\left(V_{n}\left[a_{1}, \ldots, a_{r}\right]\right) \geqslant \chi\left(V_{n}[a]\right)>n+1,
$$

a contradiction.

Suppose that $n$ is odd and $a_{1} a_{2} \cdots a_{r}>2$. Then, by Lemma 3,

$$
\chi\left(V_{n}\left[a_{1}, a_{2}, \ldots, a_{r}\right]\right) \leqslant 0,
$$

a contradiction. Q.E.D.

We remark that a similar argument shows that the signature,

$$
\tau\left(V_{2 n}\left[a_{1}, a_{2}, \ldots, a_{r}\right]\right)=1
$$

if and only if $a_{1} a_{2}, \ldots, a_{r}=1$.

\section{REFERENCES}

1. B. Y. Chen and K. Ogiue, Complete intersection manifolds with extremal Euler-Poincare characteristics, Proc. Amer. Math. Soc. 50 (1975), 121-126.

2. F. Hirzebruch, Der Satz Von Riemann-Roch in Faisceau-Theoretischer Formulierung Einige Anwendungen und Offene Fragen, Proc. Internat. Congress Math. (Amsterdam, 1954), vol. III, Noordhoff, Groningen; North-Holland, Amsterdam, 1956, pp. 457-473. MR 19, 317.

Department of Mathematics, Indiana University, Bloomington, Indiana 47401 